\title{
GLOBAL RADIOLOGICAL SOURCE SORTING, TRACKING, AND MONITORING PROJECT PHASE I FINAL REPORT
}

\author{
Randy Walker \\ David Hill \\ Bryan Gorman
}

September 2010

\author{
Prepared by \\ Oak Ridge National Laboratory \\ Oak Ridge, Tennessee 37831-6283 \\ managed by \\ UT-BATTELLE, LLC \\ for the \\ U.S. DEPARTMENT OF ENERGY \\ under contract DE-AC05-00OR22725
}





\section{CONTENTS}

Executive Overview ............................................................................................ 1

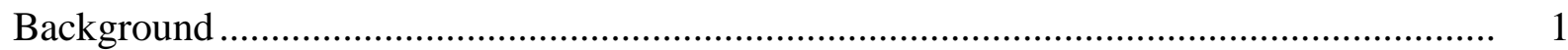

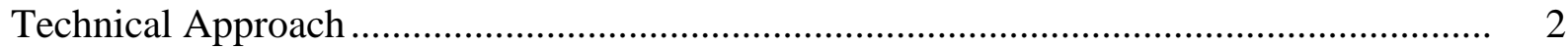

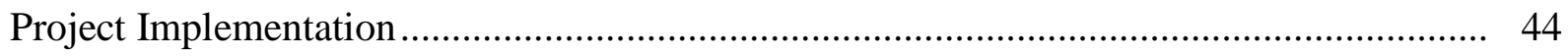

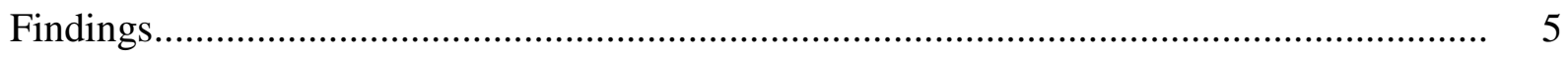

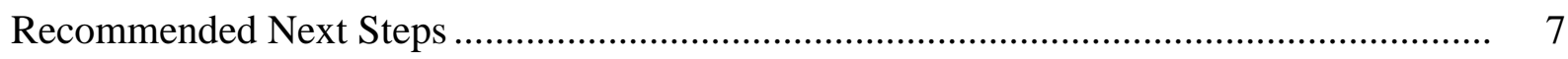

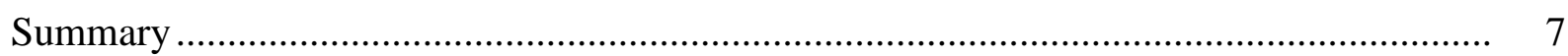

Annex A. Oak Ridge National Laboratory Shipment Lists ............................................ A-1 



\section{GLOBAL RADIOLOGICAL SOURCE SORTING, TRACKING, AND MONITORING PROJECT PHASE I FINAL REPORT}

\section{EXECUTIVE OVERVIEW}

As a proof of concept tested in an operational context, the Global Radiological Source Sorting, Tracking, and Monitoring (GRadSSTraM) Project successfully demonstrated that radio frequency identification (RFID) and Web 2.0* technologies can be deployed to track controlled shipments between the United States and the European Union. Between November 2009 and May 2010, a total of 19 shipments were successfully shipped from Oak Ridge National Laboratory (ORNL) by the U.S. Postal Service (USPS) and tracked to their delivery at England's National Physical Laboratory (NPL) by the United Kingdom Royal Mail. However, the project can only be viewed as a qualified success as notable shortcomings were observed. Although the origin and terminus of all RFID-enabled shipments were recorded and no shipments were lost, not all the waypoints between ORNL and NPL were incorporated into the pilot. Given limited resources, the project team was able to install RFID listeners/actuators at three waypoints between the two endpoints. Although it is likely that all shipments followed the same route between ORNL and NPL, it cannot be determined beyond question that all 19 shipments were routed on identical itineraries past the same three waypoints. The pilot also raises the distinct possibility that unattended RFID tracking alone, without positive confirmation that a tagged item has been properly recorded by an RFID reader, does not meet a rigorous standard for shipping controlled items. Indeed, the proof of concept test strongly suggests that a multifaceted approach to tracking may be called for, including tracking methods that are capable of reading and accepting multiple inputs for individual items [e.g., carrier-provided tracking numbers, Universal Product Codes (UPCs), and RFID tags]. For controlled items, another apparent requirement is a confirmation feature, human or otherwise, which can certify that an item's RFID tag, UPC, or tracking number has been recorded.

\section{BACKGROUND}

At a joint European Union (EU)-United States (U.S.) Summit in 2007, leaders from the U.S. Department of Commerce (DOC) and EU European Commission agreed to work together on directed "Lighthouse Priority Projects" that would "remove barriers" to transatlantic commerce. The Global Radiological Source Sorting, Tracking, and Monitoring (GRadSSTraM) pilot was one such project. Building upon the U.S. Environmental Protection Agency's (EPA's) earlier Radiological Source Tracking and Monitoring (RadSTraM) project, ${ }^{1}$ the U.S. Postal Service

\footnotetext{
${ }^{1}$ Warren T.A., et al., ORNL/TM-2008/003, RadSTraM: Radiological Source Tracking and Monitoring, Phase II Final Report, January 2007.

* According to Wikipedia, "Web 2.0 is commonly associated with web applications that facilitate interactive information sharing, interoperability, user-centered design, and collaboration on the World Wide Web. A Web 2.0 site gives its users the free choice to interact or collaborate with each other in a social media dialogue as creators (prosumer) of user-generated content in a virtual community, in contrast to websites where users (consumer) are limited to the passive viewing of content that was created for them. Examples of Web 2.0 include social-networking sites, blogs, wikis, video-sharing sites, hosted services, web applications, mashups and folksonomies."
} 
(USPS), the international Universal Postal Union, the U.S. Department of Transportation (DOT), the U.S. Department of Energy (DOE), Oak Ridge National Laboratory (ORNL), EPA, and EU collaborated to "develop a joint framework for cooperation on identification and development of best practices for radio frequency identification (RFID) technologies." The goal of the pilot was to track commercial shipments of radiological isotopes between the United States and EU.

\section{TECHNICAL APPROACH}

In July 2009, a team of collaborators produced a concept of operations (ConOps) plan $^{2}$ for Phase I of the GRadSSTraM pilot. The following objectives were cited for the pilot.

1. Validate the performance of Web 2.0 enabled RFID tracking systems to monitor Express Mail Service (EMS) shipments of radioisotopes in the international supply chain.

2. Quantify the reliability of these tracking systems with regard to probability of tag detection and operational reliability at checkpoints and choke points in the supply chain process network.

3. Determine whether implementation of these systems will help to reduce regulatory burden and enhance transatlantic trade.

4. Demonstrate that RFID tracking and monitoring of radioactive materials is ready for Phase II testing using commercial isotope shippers and carriers.

5. Establish a prototype Web 2.0 site to enhance the use of tracking technologies internationally.

To address these objectives, the ConOps document prescribed a proof of concept test involving deployment of an RFID tracking network to monitor radioisotope shipments between ORNL and the National Physical Laboratory (NPL) in Teddington, United Kingdom (UK). The progress of the shipments would be displayed on a Web 2.0 Google Maps mashup (Figure 1) identifying the location of each shipment as it progressed through its route and was scanned by the prepositioned RFID listeners/actuators.

Early in the implementation of the plan, adjustments were required to keep the project on budget and to reduce any safety concerns for the participants. The original operational requirements (followed by qualifying notes indicating actual practice as a result of the required adjustments) included the following:

1. Limited quantity (Ltd Qty) radioactive material; RFID modified inner containers with electronic seal.

NOTE: Because of safety concerns, radioactive materials were eliminated from the shipments, and surrogate containers were shipped and tracked.

\footnotetext{
${ }^{2}$ Walker R. M., et al., Concept of Operations Plan, July 2009.
} 
2. USPS and Royal Mail designated strong tight containers per the applicable code or regulations.

NOTE: Certified isotope shipping containers were substituted with boxes to reduce costs and losses.

3. Ten round-trip Ltd Qty shipments between ORNL and NPL.

NOTE: A total of 19 shipments, all originating at ORNL and terminating at NPL, were tracked during the pilot.

4. Packaging configured with active, passive, or hybrid RFID tags or a combination thereof.

NOTE: Because resources were limited, USPS provided passive RFID tags.

5. Testing configuration and RFID listeners/actuators installed at ORNL, NPL, and at designated USPS/Royal Mail terminal and distribution facilities.

NOTE: RFID listeners/actuators were installed at: ORNL; the Knoxville Post Office at Middlebrook Pike and Weisgarber Road; Miami International and Heathrow (London) Airports; and NPL.

6. A Web 2.0 data collection and distribution engine for disparate and distributed data integrated with ORNL, NPL, USPS, and Royal Mail process networks.

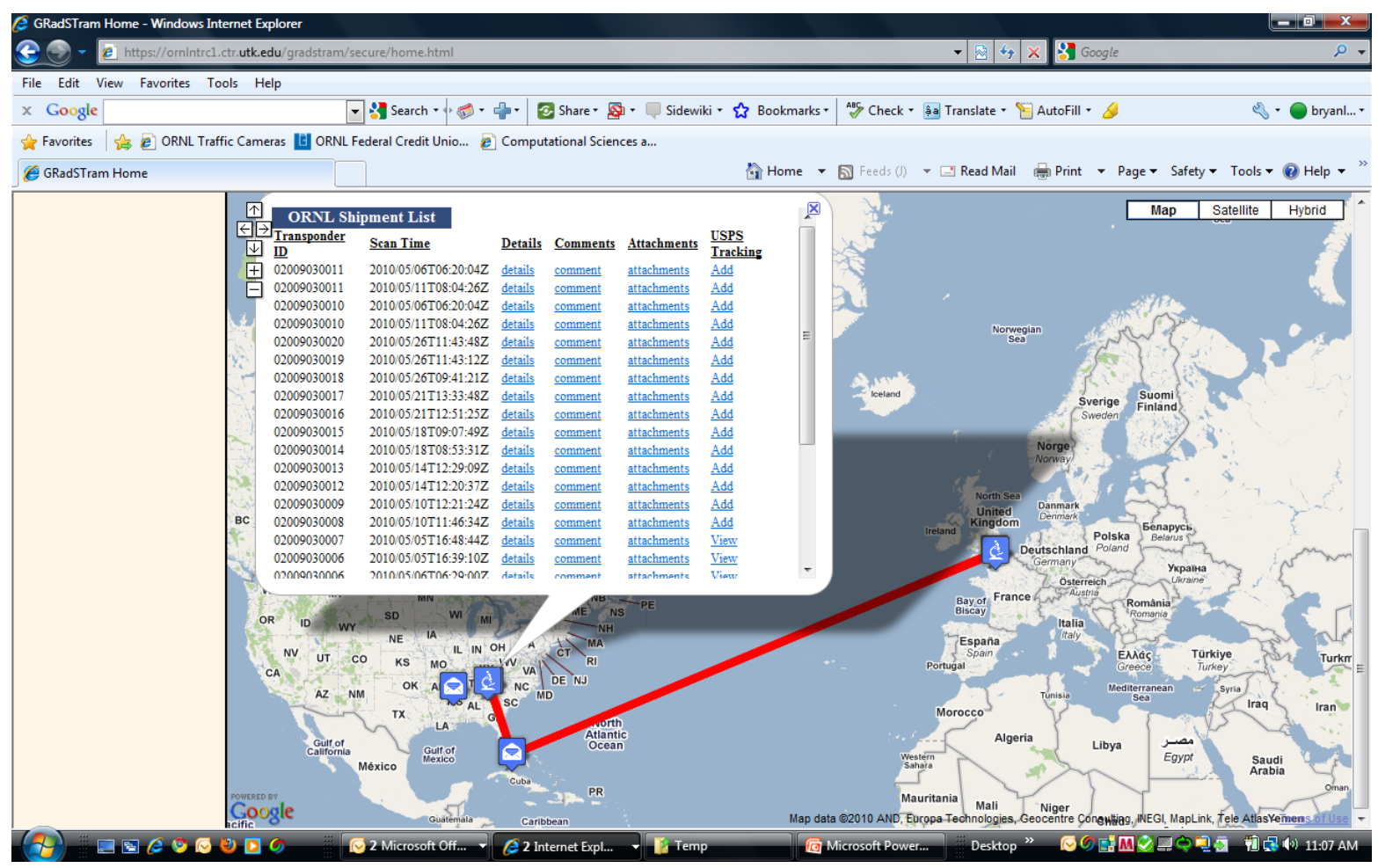

Figure 1. ORNL GRadSSTraM shipments to NPL. 


\section{PROJECT IMPLEMENTATION}

Between November 2009 and May 2010, a total of 19 shipments were routed from ORNL to NPL (see Annex A, “ORNL Shipment List").

1. All 19 shipments were tagged at ORNL, and the origin of the shipment was scanned and recorded on the GRadSSTraM Web site.

2. All 19 shipments were scanned at the receiving terminus at NPL, and their arrival was scanned and recorded on the GRadSSTraM site.

3. Manual entries at Knoxville (Figure 2) were posted for two shipments (02009030002, 02009030003).

4. Digitized copies of the ORNL-19 shipping form (Figure 3) were accompanied with (scanned and recorded on the GRadSSTraM site.

5. Four of the 19 shipments (02009030002, 02009030003, 02009030012, and 02009030013) were tracked and recorded through Heathrow.

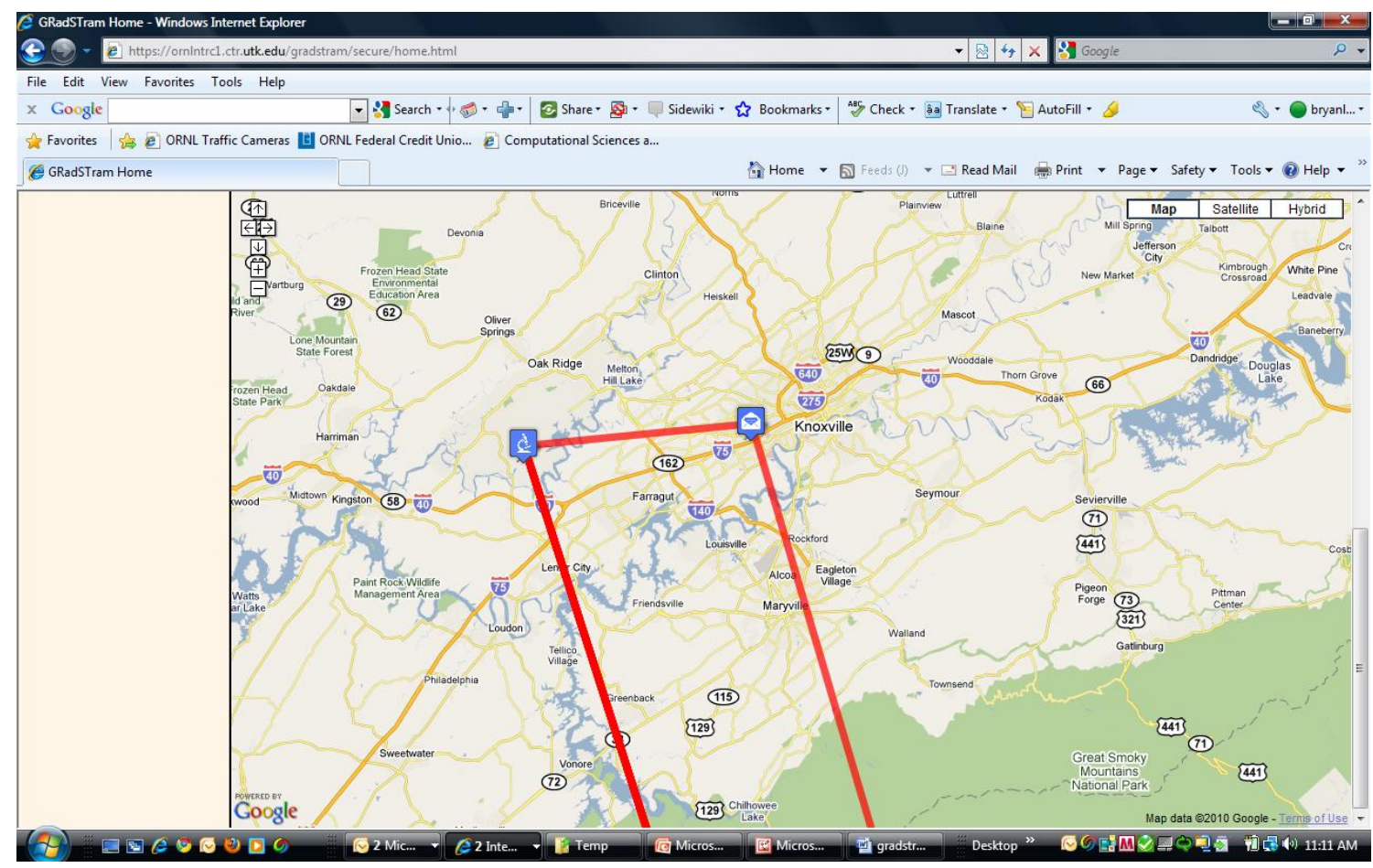

Figure 2. Manual entries between ORNL and Knoxville. 


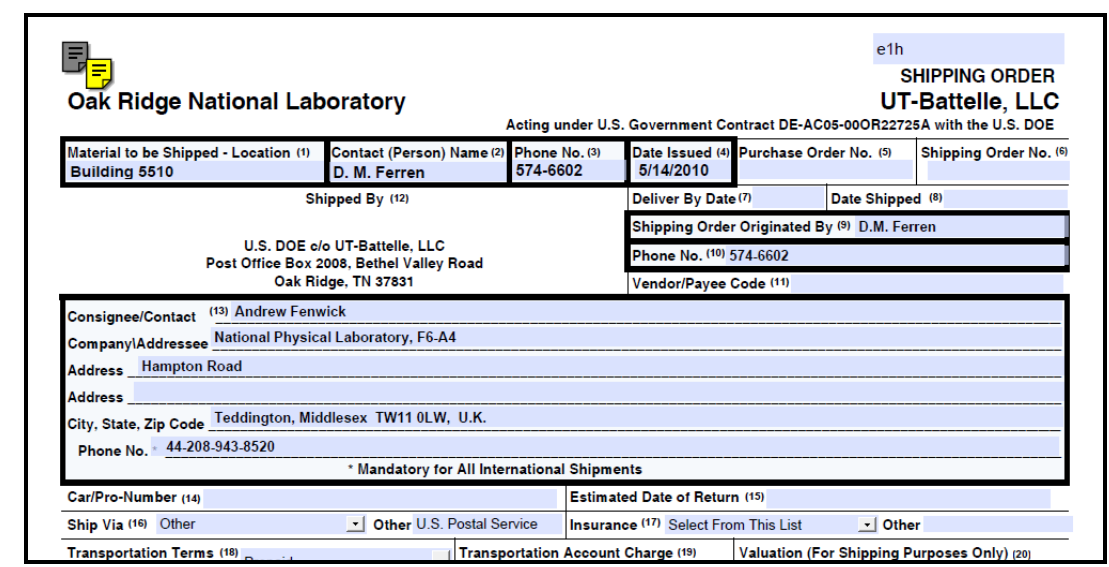

Figure 3. ORNL shipping form (ORNL-19).

\section{FINDINGS}

1. The Knoxville Post Office RFID listener/actuator, which was a passive device, did not record any of the 19 ORNL shipments. Owing to extremely limited resources, rigorous efforts to troubleshoot the system to determine the cause (hardware, installation issues, absence of local support) for the failure could not be conducted.

2. The USPS contracts with FedEx to transport bulk shipments between Knoxville and Miami. FedEx uses its own enterprise tracking system. Resources did not exist to deploy an RFID listening device in FedEx distribution centers in Knoxville, Memphis, or Miami. Although the 19 shipments were shipped between Knoxville and Miami through Memphis, no record of the Memphis waypoint exists. However, given the resources, it would be quite possible to extend the GRadSSTraM interface to the FedEx system and eliminate the need for the additional RFID listeners/actuators by relying instead on the FedEx enterprise tracking system.

3. The Heathrow RFID listener/actuator picked up 4 of the 19 shipments. At this point, we don't have sufficient information to determine why the other 15 shipments were not tracked or recorded through Heathrow. Possible reasons that have been conjectured include:

- the RFID reader at Heathrow may have been at a location the shipments did not pass,

- the reader may not have been operational at all times, and

- $\quad$ the shipments may have passed through an alternative airport or carrier

4. Although the ORNL shipments to NPL were not automatically recorded by the RFID scanner at the Knoxville Post Office, the GRadSSTraM web site allowed for users to manually record shipments. If USPS access to and use of the GRadSSTraM web site had been exploited at the Knoxville office, the GRadSSTraM shipments through there could have been recorded manually. Moreover, the internal FedEx tracking number that was assigned to the shipment between Knoxville and Miami could have been annotated, permitting the web site to track the shipment through the FedEx distribution points. 
5. Although the origin and terminus of all RFID-enabled shipments were recorded and no shipments were lost, not all the waypoints between ORNL and NPL were recorded for each shipment. At various times, RFID sensors at intervening waypoints did not record and report shipments. At Miami, for example, where there were two readers, there were some shipments that were "read" by one of the listeners/actuators (sometimes several times) but not by the other. Although the reasons for the missing tracks cannot be explained in every case, certain tentative conclusions can be drawn from the circumstances. The most likely explanation is that a tagged item was not scanned because it did not come within range of the listener/actuator. It is also possible, although perhaps less likely, that a particular shipment could have been routed through a different path out of the range of the reader. In the cases of the Knoxville station and the 15 shipments that were not "read" at Heathrow, there is the possibility that the readers were not operational. At Heathrow, there is also the unlikely possibility that the 15 items were not routed through the airport-unlikely because most shipments from Miami to England are routed through Heathrow. In general, operator training in the use of GRadSSTraM, as well as awareness of its capabilities, would have improved access to alternative tracking information along a particular shipment's route. The pilot, given limited resources, was deployed at only three known waypoints between the two known endpoints, and it is not clear whether all 19 shipments followed identical itineraries. The pilot also raises the distinct possibility that unattended RFID tracking alone, without positive confirmation that a tagged item has been properly recorded by an RFID reader, does not meet a rigorous standard for shipping controlled items. Indeed, this proof of concept test strongly suggests the need for a multifaceted approach to tracking such as a tracking tool that is capable of reading and accepting multiple tracking inputs [e.g., carrier-provided tracking numbers, Universal Product Codes (UPCs), and RFID tags]. For controlled items, there is a concurrent requirement for a confirmation feature, human or otherwise, which can certify that an item's RFID tag, UPC, or tracking number has been recorded.

The following are the specific findings for the objectives outlined under the technical approach.

1. Validate the performance of Web 2.0 enabled RFID tracking systems to monitor Express Post shipments of radioisotopes in the international supply chain.

The Phase I effort was a qualified success. Clearly the Web 2.0 Google Maps mashup was able to automatically display the RFID annotations and also display any annotations that were manually posted to the shipment along the way (e.g., the ORNL-19 forms). The GRadSSTraM Web 2.0 tracking tool offers great potential as a multifaceted tracking tool that can bridge different legacy and emerging technologies. Continued development and testing, with end-user participation in future pilots, is strongly recommended.

2. Quantify the reliability of these tracking systems with regards to probability of tag detection and operational reliability at checkpoints and choke points in the supply chain process network.

The RFID listeners/actuators worked $100 \%$ of the time at ORNL and NPL. At those two locations, there were operators who confirmed that the tag was "read." At Miami, where two readers were deployed, all 19 shipments were recorded by at least one reader and reported to the Google Maps mashup. At Heathrow, where one reader was deployed, 4 of the 19 shipments 
were recorded and reported. Additional methods, using either existing tracking capabilities, redundant sensors, or new external checks, are necessary before an unattended RFID sensor can be relied upon to track a controlled item with $100 \%$ assurance.

3. Determine whether implementation of these systems will help to reduce regulatory burden and enhance transatlantic trade.

The current pilot did not provide data to suggest that either RFID or Web 2.0 technologies alone will reduce regulations or streamline processes for securing transatlantic trade. However, there is the potential that these two emerging technologies, combined with operational methods and practices and links to enterprise tracking systems, can improve the security of shipments and, thereby, increase the enforcement of regulations and, ultimately, reduce loss and improve accountability in the commercial networks.

4. Demonstrate that RFID tracking and monitoring of radioactive materials is ready for Phase II testing using commercial isotope shippers and carriers.

The results of Phase I, which was conducted with minimal funding, demonstrate that both RFID and emerging Web 2.0 technologies have future roles in securing the supply chain.

5. Establish a prototype standard architecture to enhance the use of tracking technologies internationally.

While there is insufficient evidence to specify a standard future architecture for tracking shipments, there is ample evidence to suggest that such an architecture will be heterogeneous and not homogeneous. Some aspects of shipping will continue to be manually logged; others will be tracked by legacy enterprise solutions (e.g., scanners and UPCs). Still newer technologies (e.g., Global Positioning System, social media, and social networks) will be a part of the supply chain future.

\section{RECOMMENDED NEXT STEPS}

A broader and more robust deployment of Web 2.0 and RFID technologies, with the active participation of the public and private practitioners involved in the supply chain, is justified and will likely result in moving these nascent technologies closer to adoption.

\section{SUMMARY}

As a proof of concept tested in an operational context, Phase I of GRadSSTraM demonstrated that RFID and Web 2.0 technologies can be deployed to track controlled shipments between the United States and EU. Between November 2009 and May 2010, a total of 19 shipments were successfully shipped from ORNL by USPS and tracked to their delivery at NPL by the UK Royal Mail. Although the efficacy of RFID and Web 2.0 technologies for international tracking was demonstrated, Phase I can only be viewed as a preliminary and qualified success; notable shortcomings were observed. For example, the origin and terminus of all RFID-enabled 
shipments were recorded and no shipments were lost; however, not all the waypoints between ORNL and NPL were incorporated into the pilot. Given limited resources, the project team was able to install RFID listeners/actuators at three waypoints between the two endpoints. Although it is likely that all shipments followed the same route between ORNL and NPL, it cannot be determined beyond question that all 19 shipments were routed on identical itineraries past the same three waypoints. The pilot also raises the distinct possibility that unattended RFID tracking alone, without positive confirmation that a tagged item has been properly recorded by an RFID reader, does not meet a rigorous standard for shipping controlled items. This proof of concept test strongly suggests the need for a multifaceted approach to tracking such as a tracking method that is capable of reading and accepting multiple tracking inputs (e.g., carrier-provided tracking numbers, UPCs, and RFID tags). For controlled items, another apparent requirement is a confirmation feature, human or otherwise, which can substantiate that an item's RFID tag, UPC, or tracking number has been recorded. 



\section{ANNEX A. OAK RIDGE NATIONAL LABORATORY SHIPMENT LISTS}

\section{Shipment 1: 02009030002}

ORNL 2009/10/28T14:50:28Z

ORNL 2009/11/04T09:27:11Z

* Knoxville 2009/11/05T03:05:00Z This is a manual record

Miami 2009/11/05T07:54:00Z

Miami 2009/11/05T07:56:00Z

Miami 2009/11/05T08:43:00Z

Miami 2009/11/05T08:50:00Z

Miami 2009/11/05T11:19:21Z

Miami 2009/11/05T12:29:42Z

London Heathrow 2009/11/06T10:45:00Z

NPL 2009/11/09T14:46:31Z

NPL 2009/11/09T15:21:08Z

Shipment 2: 02009030003

ORNL 2009/11/10T09:55:58Z

* Knoxville 2009/11/11T02:45:00Z This is a manual entry. Miami 2009/11/11T08:32:00Z

Miami 2009/11/11T09:28:00Z

Miami 2009/11/11T09:29:00Z

London Heathrow 2009/11/12T11:07:00Z

NPL 2009/11/17T08:17:28Z

\section{Shipment 3: 02009030005}

ORNL-19 shipping form http://home.ornl.gov/ webworks/ORNL/ORNL-

19/ship_5_3_110:15541.fdf

Customs Declaration No. CJ 048897131 US ORNL Ship Order No. X152875 Shipped $5 / 4 / 2010$

ORNL 2010/04/28T13:50:25Z

ORNL 2010/05/03T14:00:48Z

Miami 2010/05/06T08:04:00Z

Miami 2010/05/06T08:05:00Z

Miami 2010/05/06T08:06:00Z

Miami 2010/05/06T10:30:00Z

Miami 2010/05/06T10:30:32Z

Miami 2010/05/06T10:31:00Z

Miami 2010/05/06T11:08:00Z

Miami 2010/05/06T11:09:00Z

Miami 2010/05/06T11:12:00Z

Miami 2010/05/06T11:12:54Z 
Miami 2010/05/06T11:13:00Z

Miami 2010/05/06T11:33:00Z

Miami 2010/05/06T11:33:19Z

Miami 2010/05/06T11:34:00Z

Miami 2010/05/06T12:15:19Z

Miami 2010/05/06T12:41:22Z

Miami 2010/05/06T13:21:45Z

Miami 2010/05/06T13:27:42Z

NPL 2010/05/11T14:05:02Z

\section{Shipment 4: 02009030004}

ORNL-19 shipping form http://home.ornl.gov/ webworks/ORNL/ORNL-

19/ship_5_3_110:14584.fdf

Customs Declaration No. CJ 048956373 US ORNL Ship Order No. X152896 Shipped 5/4/2010

ORNL 2010/05/03T13:48:40Z

ORNL 2010/05/04T07:14:59Z

Miami 2010/05/06T10:34:00Z

Miami 2010/05/06T10:34:09Z

Miami 2010/05/06T10:35:00Z

Miami 2010/05/06T12:15:53Z

NPL 2010/05/11T14:05:01Z

\section{Shipment 5: 02009030006}

ORNL-19 shipping form http://home.ornl.gov/ webworks/ORNL/ORNL-

19/ship 5 5 110:17485.fdf

Customs Declaration No. CJ 049111695 US. ORNL Ship Order X152959, Shipped 5/7/10. Error in previous entry: Customs Declaration No. CJ 049108475 US, ORNL Shipping Order No. X152957, shipped 5/7/10.

ORNL 2010/05/05T16:39:10Z ORNL 2010/05/06T06:29:00Z Miami 2010/05/08T08:40:00Z Miami 2010/05/08T08:40:51Z Miami 2010/05/08T08:41:00Z Miami 2010/05/08T08:42:00Z Miami 2010/05/08T09:11:00Z Miami 2010/05/08T09:11:11Z Miami 2010/05/08T09:12:00Z Miami 2010/05/08T10:46:00Z Miami 2010/05/08T10:46:36Z Miami 2010/05/08T10:47:00Z Miami 2010/05/08T12:21:00Z Miami 2010/05/08T12:22:00Z 
Miami 2010/05/08T12:22:11Z

Miami 2010/05/08T12:23:00Z

NPL 2010/05/10T10:50:24Z

\section{Shipment 6: 02009030007}

ORNL-19 shipping form http://home.ornl.gov/ webworks/ORNL/ORNL-

19/ship_5_5_110:175653.fdf

Customs Declaration No. CJ 049111695 US, ORNL Shipping Order No. X152959, shipped $5 / 7 / 10$.

ORNL 2010/05/05T16:48:44Z

Miami 2010/05/08T08:41:00Z

Miami 2010/05/08T08:41:16Z

Miami 2010/05/08T08:42:00Z

Miami 2010/05/08T09:11:00Z

Miami 2010/05/08T09:11:11Z

Miami 2010/05/08T09:12:00Z

Miami 2010/05/08T10:46:00Z

Miami 2010/05/08T10:47:00Z

Miami 2010/05/08T10:47:07Z

Miami 2010/05/08T10:48:00Z

Miami 2010/05/08T10:50:00Z

Miami 2010/05/08T10:50:15Z

Miami 2010/05/08T10:51:00Z

NPL 2010/05/10T10:50:23Z

\section{Shipment 7: 02009030008}

ORNL-19 shipping form http://home.ornl.gov/ webworks/ORNL/ORNL-

19/ship_5_10_110:125050.fdf

Customs Declaration CJ 049342045 US ORNL Ship Order No. X153061 Shipped 5/11/2010

ORNL 2010/05/10T11:46:34Z

Miami 2010/05/12T07:56:00Z

Miami 2010/05/12T07:56:08Z

Miami 2010/05/12T10:28:00Z

Miami 2010/05/12T10:28:10Z

Miami 2010/05/12T11:42:00Z

Miami 2010/05/12T11:42:46Z

Miami 2010/05/12T11:43:00Z

NPL 2010/05/17T13:22:02Z 


\section{Shipment 8: 02009030009}

ORNL-19 shipping form http://home.ornl.gov/ webworks/ORNL/ORNL-

19/ship_5_10_110:132237.fdf

Customs Declaration No. CJ 049345761 US ORNL Ship Order No. X153062 Shipped $5 / 11 / 2010$

ORNL 2010/05/10T12:21:24Z

Miami 2010/05/12T07:56:00Z

Miami 2010/05/12T07:56:08Z

Miami 2010/05/12T10:25:00Z

Miami 2010/05/12T10:25:25Z

Miami 2010/05/12T10:26:00Z

Miami 2010/05/12T11:09:00Z

Miami 2010/05/12T11:09:51Z

Miami 2010/05/12T11:10:00Z

Miami 2010/05/12T11:42:00Z

Miami 2010/05/12T11:42:45Z

Miami 2010/05/12T11:43:00Z

Miami 2010/05/12T12:09:06Z

NPL 2010/05/17T13:22:02Z

\section{Shipment 9: 02009030010}

Customs Declaration No. CJ 049480506 US ORNL Ship Order No. X153063 Shipped $5 / 13 / 2010$

ORNL 2010/05/06T06:20:04Z

ORNL 2010/05/11T08:04:26Z

Miami 2010/05/14T08:31:00Z

Miami 2010/05/14T08:31:36Z

Miami 2010/05/14T08:32:00Z

Miami 2010/05/14T10:47:00Z

Miami 2010/05/14T10:47:19Z

Miami 2010/05/14T10:48:00Z

Miami 2010/05/14T10:52:00Z

Miami 2010/05/14T10:52:47Z

Miami 2010/05/14T10:53:00Z

Miami 2010/05/14T11:31:00Z

Miami 2010/05/14T11:31:50Z

Miami 2010/05/14T11:32:00Z

Miami 2010/05/14T12:35:33Z

NPL 2010/05/17T13:22:02Z 


\section{Shipment 10: 02009030011}

Customs Declaration No. CJ 049481135 US ORNL Ship Order No. X153064 Shipped $5 / 13 / 2010$

ORNL 2010/05/06T06:20:04Z

ORNL 2010/05/11T08:04:26Z

Miami 2010/05/14T08:31:00Z

Miami 2010/05/14T08:31:35Z

Miami 2010/05/14T08:32:00Z

Miami 2010/05/14T10:43:00Z

Miami 2010/05/14T10:44:00Z

Miami 2010/05/14T10:44:05Z

Miami 2010/05/14T11:31:00Z

Miami 2010/05/14T11:31:48Z

Miami 2010/05/14T11:32:00Z

Miami 2010/05/14T12:35:04Z

NPL 2010/05/17T13:22:03Z

\section{Shipment 11: 02009030012}

ORNL-19 shipping form http://home.ornl.gov/ webworks/ORNL/ORNL-

19/ship $5 \quad 14 \quad 110: 132241$. fdf

Customs Declaration No. CJ 049789408 US ORNL Ship Order No. X153113 Shipped $5 / 18 / 2010$

ORNL 2010/05/14T12:20:37Z

Miami 2010/05/19T07:53:00Z

Miami 2010/05/19T07:53:28Z

Miami 2010/05/19T07:54:00Z

Miami 2010/05/19T10:29:00Z

Miami 2010/05/19T10:29:56Z

Miami 2010/05/19T10:30:00Z

Miami 2010/05/19T12:06:23Z

London Heathrow 2010/05/20T08:34:00Z

NPL 2010/05/21T15:36:35Z

\section{Shipment 12: 02009030013}

ORNL-19 shipping form http://home.ornl.gov/ webworks/ORNL/ORNL-

19/ship_5_14_110:133117.fdf

Customs Declaration No. CJ 049792526 US ORNL Ship Order No. X153114 Shipped $5 / 18 / 2010$

ORNL 2010/05/14T12:29:09Z

Miami 2010/05/19T07:56:00Z 
Miami 2010/05/19T07:56:14Z

Miami 2010/05/19T07:57:00Z

Miami 2010/05/19T10:31:00Z

Miami 2010/05/19T10:31:39Z

Miami 2010/05/19T10:32:00Z

Miami 2010/05/19T11:12:00Z

Miami 2010/05/19T11:12:24Z

Miami 2010/05/19T12:06:23Z

London Heathrow 2010/05/20T08:35:00Z

NPL 2010/05/21T15:36:35Z

\section{Shipment 13: 02009030014}

ORNL-19 shipping form http://home.ornl.gov/ webworks/ORNL/ORNL-

19/ship_5_18_110:95539.fdf

Customs Declaration No. CJ 049965703 US ORNL Ship Order No. X153233 Shipped $5 / 20 / 2010$

ORNL 2010/05/18T08:53:31Z

Miami 2010/05/21T10:28:00Z

Miami 2010/05/21T10:28:33Z

Miami 2010/05/21T10:29:00Z

NPL 2010/05/24T10:33:31Z

\section{Shipment 14: 02009030015}

ORNL-19 shipping form http://home.ornl.gov/ webworks/ORNL/ORNL-

19/ship_5_18_110:101239.fdf

Customs Declaration No. CJ 049967620 US ORNL Ship Order No. X153232 Shipped $5 / 20 / 2010$

ORNL 2010/05/18T09:07:49Z

Miami 2010/05/21T10:27:00Z

Miami 2010/05/21T10:27:13Z

Miami 2010/05/21T10:28:00Z

NPL 2010/05/24T10:33:31Z

\section{Shipment 15: 02009030016}

ORNL-19 shipping form http://home.ornl.gov/ webworks/ORNL/ORNL-

19/ship_5_21_110:135155.fdf

Customs Declaration No. CJ 000196965 US ORNL Ship Order No. X153379 Shipped $5 / 25 / 2010$

ORNL 2010/05/21T12:51:25Z

Miami 2010/05/26T10:21:00Z 
Miami 2010/05/26T10:21:56Z

Miami 2010/05/26T10:22:00Z

Miami 2010/05/26T10:26:00Z

Miami 2010/05/26T10:27:00Z

Miami 2010/05/26T11:24:00Z

Miami 2010/05/26T11:25:00Z

Miami 2010/05/26T11:25:08Z

Miami 2010/05/26T11:26:00Z

Miami 2010/05/26T12:03:27Z

Miami 2010/05/26T14:10:02Z

Miami 2010/05/26T14:10:53Z

Miami 2010/05/26T14:22:53Z

Miami 2010/05/26T14:23:43Z

Miami 2010/05/26T14:35:17Z

Miami 2010/05/26T14:36:07Z

Miami 2010/05/26T14:50:07Z

Miami 2010/05/26T14:50:55Z

Miami 2010/05/26T15:00:33Z

Miami 2010/05/26T15:01:32Z

Miami 2010/05/26T15:05:45Z

Miami 2010/05/26T15:05:53Z

Miami 2010/05/26T15:06:42Z

Miami 2010/05/26T15:18:23Z

Miami 2010/05/26T15:19:12Z

Miami 2010/05/26T15:31:33Z

Miami 2010/05/26T15:32:22Z

NPL 2010/06/07T07:32:00Z

\section{Shipment 16: 02009030017}

ORNL-19 shipping form http://home.ornl.gov/ webworks/ORNL/ORNL-

19/ship_5_21_110:144232.fdf

Customs Declaration No. CJ 000197719 US ORNL Ship Order No. X153380 Shipped $5 / 25 / 2010$

ORNL 2010/05/21T13:33:48Z

Miami 2010/05/26T07:31:00Z

Miami 2010/05/26T07:32:00Z

Miami 2010/05/26T07:32:04Z

Miami 2010/05/26T09:45:00Z

Miami 2010/05/26T09:45:32Z

Miami 2010/05/26T09:46:00Z

Miami 2010/05/26T10:19:00Z

Miami 2010/05/26T10:19:40Z

Miami 2010/05/26T10:20:00Z

Miami 2010/05/26T11:09:00Z 
Miami 2010/05/26T11:10:00Z

Miami 2010/05/26T11:10:12Z

Miami 2010/05/26T11:11:00Z

Miami 2010/05/26T11:24:00Z

Miami 2010/05/26T11:25:00Z

Miami 2010/05/26T11:25:14Z

Miami 2010/05/26T11:26:00Z

Miami 2010/05/26T12:03:33Z

NPL 2010/06/07T07:32:00Z

\section{Shipment 17: 02009030018}

ORNL-19 shipping form http://home.ornl.gov/ webworks/ORNL/ORNL-

19/ship 5 26 110:104017.fdf

Customs Declaration No. CJ 001397040 US, ORNL Order No. X153669, Shipped 6/14/2010.

ORNL 2010/05/26T09:41:21Z

Miami 2010/06/15T10:33:00Z

Miami 2010/06/15T10:33:31Z

Miami 2010/06/15T10:34:00Z

Miami 2010/06/15T11:24:00Z

Miami 2010/06/15T11:24:45Z

Miami 2010/06/15T11:25:00Z

NPL 2010/06/17T14:57:53Z

\section{Shipment 18: 02009030019}

ORNL-19 shipping form http://home.ornl.gov/ webworks/ORNL/ORNL-

19/ship_5_26_110:104242.fdf

Customs Declaration No. CJ 001398924 US, ORNL Ship Order No. X153670, shipped $6 / 14 / 2010$.

ORNL 2010/05/26T11:43:12Z

Miami 2010/06/15T10:36:59Z

Miami 2010/06/15T10:37:00Z

Miami 2010/06/15T11:14:00Z

Miami 2010/06/15T11:14:39Z

Miami 2010/06/15T11:15:00Z

Miami 2010/06/15T11:24:00Z

Miami 2010/06/15T11:24:42Z

Miami 2010/06/15T11:25:00Z

Miami 2010/06/15T12:14:09Z

Miami 2010/06/15T13:11:53Z

NPL 2010/06/17T14:57:53Z 


\section{Shipment 19: 02009030020}

ORNL-19 shipping form http://home.ornl.gov/ webworks/ORNL/ORNL-

19/ship_5_26_110:10440.fdf

Customs Declaration No. CJ 001399981 US, ORNL Ship Order No. X153671, shipped $6 / 14 / 2010$.

ORNL 2010/05/26T11:43:48Z

Miami 2010/06/15T07:53:00Z

Miami 2010/06/15T07:53:17Z

Miami 2010/06/15T07:54:00Z

Miami 2010/06/15T10:33:00Z

Miami 2010/06/15T10:33:09Z

Miami 2010/06/15T10:34:00Z

Miami 2010/06/15T10:36:00Z

Miami 2010/06/15T10:36:19Z

Miami 2010/06/15T10:37:00Z

Miami 2010/06/15T11:24:00Z

Miami 2010/06/15T11:24:43Z

Miami 2010/06/15T11:25:00Z

Miami 2010/06/15T12:14:17Z

NPL 2010/06/17T14:57:53Z 